\title{
Embedding If and Only If
}

\author{
Adam Sennet • Jonathan Weisberg
}

Received: 24 April 2010 / Accepted: 12 January 2011 / Published online: 1 February 2011

(C) The Author(s) 2011. This article is published with open access at Springerlink.com

\begin{abstract}
Some left-nested indicative conditionals are hard to interpret while others seem fine. Some proponents of the view that indicative conditionals have No Truth Values (NTV) use their view to explain why some left-nestings are hard to interpret: the embedded conditional does not express the truth conditions needed by the embedding conditional. Left-nestings that seem fine are then explained away as cases of ad hoc, pragmatic interpretation. We challenge this explanation. The standard reasons for NTV about indicative conditionals (triviality results, Gibbardian standoffs, etc.) extend naturally to NTV about biconditionals. So NTVers about conditionals should also be NTVers about biconditionals. But biconditionals embed much more freely than conditionals. If NTV explains why some left-nested conditionals are hard to interpret, why do biconditionals embed successfully in the very contexts where conditionals do not embed?
\end{abstract}

Keywords Conditionals · Triviality results

\footnotetext{
A. Sennet

Department of Philosophy, University of California, Davis, 1 Shields Avenue,

Davis, CA 95616, USA

e-mail: amsennet@ucdavis.edu

J. Weisberg $(\bowtie)$

Department of Philosophy, University of Toronto, 170 St. George Street, Rm. 516, Toronto, ON M5R 2M8, Canada

e-mail: jonathan.weisberg@utoronto.ca
} 


\section{Introduction}

Some left-nested indicative conditionals are hard to interpret while others seem fine. ${ }^{1}$ Gibbard [6] famously offers the following, said of a conference, as an example of troublesome left-nesting:

(1) *If Kripke was there if Strawson was there, Anscombe was there. ${ }^{2}$

In contrast, the following example seems unproblematic:

(2) If the cup broke if it was dropped, it was fragile.

The difference between (1) and (2) poses an explanatory puzzle, one we might expect our semantic theory of indicative conditionals to solve.

One prominent view is that indicative conditionals do not have truth values (NTV) because they do not express propositions. When I say that if Sergio makes coffee then he'll spill some, I may express an epistemic attitude or conditionally assert something, but I do not assert anything unconditionally that can properly be evaluated for truth or falsity. According to some proponents of this view, like Gibbard [6], Edgington [5] and Bennett [2], (1) is uninterpretable because the nested conditional Kripke was there if Strawson was there does not express truth conditions in a context where they are expected, and the result is semantic defectiveness. While the nested the cup broke if it was dropped likewise fails to express truth conditions where expected, (2) can still be interpreted by ad hoc, pragmatic means. When we hear (2), we substitute for the embedded conditional a factual basis that would make the embedded conditional assertable, e.g. the cup was disposed to break upon falling. So when we hear (2), we understand it as saying something to the effect of:

(3) If the cup was disposed to break upon falling, it was fragile.

On this view, the default expectation is that a left-nested conditional will not be interpretable, but pragmatics can salvage it when an ad hoc interpretation is available.

We want to challenge this way of handling the explanatory puzzle posed by (1) and (2). The crux of our challenge will be that the standard reasons to hold NTV about conditionals are likewise reasons to hold NTV about biconditionals, yet biconditionals embed much more freely than conditionals. In particular, if and only if embeds successfully in the very contexts where embedding if/then makes for apparent uninterpretability. Since NTVers should be equally NTVist about if and only if, they should expect if and only if to

\footnotetext{
${ }^{1}$ The right-nested form $A \rightarrow(B \rightarrow C)$ is typically thought not to exhibit this puzzling phenomenon, and so will not be discussed.

${ }^{2}$ Notice that interpretability is not helped if we move to the if if $A$ then $B$, then $C$ formulation, instead of the if $A$ if $B$, then $C$ formulation that Gibbard uses. We will follow Gibbard and others in focusing on the if $A$ if $B$, then $C$ formulation here and in subsequent examples. Readers who are concerned that something may hang on this choice should check to their satisfaction that there is no significant difference in each instance.
} 
display a similar pattern of (un)interpretability. So why does if and only if embed where if/then does not?

A clarificatory note before we begin: some NTVers endorse theories that do freely allow left-nesting. ${ }^{3}$ These NTVers will presumably attribute (1)'s difficulty to something besides a failure to have truth conditions where expected, so they are not our target. Our target is not the NTV view itself but, rather, the use of NTV to explain (1)'s difficulty.

We will proceed as follows. Section 2 reviews some well-known arguments for NTV about conditionals and shows how they extend to biconditionals. Section 3 argues that if and only if embeds much more freely than iffthen; most importantly, it embeds in the very same sentences where if/then does not embed. Section 4 closes with a brief discussion of the import of these results.

\section{Arguments for NTV}

Why think that indicative conditionals do not have truth values? Many reasons have been given but we will focus on two: Lewis's triviality result and Gibbardian standoffs. In each case, we claim, the argument readily extends to biconditionals. We will also briefly consider how two further arguments, due to Edgington and Bradley, can be similarly extended. In all four cases we will treat the original assumptions and steps uncritically, showing only how they extend to the case of biconditionals.

Lewis [10] showed that the probability of a conditional $A \rightarrow B$ is always trivialized, given the plausible assumption that the probability of a conditional is always the same as the corresponding conditional probability. Following Edgington, we label this assumption: ${ }^{4}$

The Equation $p(A \rightarrow B)=p(B \mid A)$ for any $A$ and $B$.

Given The Equation, it follows that $p(A \rightarrow B)=p(B)$ for any $A$ and $B$, which is absurd. But this result can be blocked if we accept NTV. If $A \rightarrow B$ does not express a proposition, then $p(A \rightarrow B)$ is not the probability of a proposition to which the laws of probability can be applied. Instead, $p(A \rightarrow B)$ is the acceptability of the conditional $A \rightarrow B .^{5}$ The triviality result thus supports NTV, since NTV allows us to avoid absurdity while retaining The Equation.

To extend the argument to biconditionals we must add to The Equation some further assumption about the probabilities of biconditionals. There are several ways to go here.

\footnotetext{
${ }^{3}$ See [1], [7], and [9].

${ }^{4}$ The class of probability functions over which $p$ ranges is assumed, here and in the remainder of our discussion of triviality results, to be closed under conditioning.

${ }^{5}$ Some interpret The Equation as presupposing that $A \rightarrow B$ expresses a proposition. For them the NTV response is framed differently: The Equation is rejected, but its appeal is preserved in the thesis that the assertability of a conditional matches the corresponding conditional probability.
} 
One natural way to go is to assume that the probability of a biconditional is always the probability of a conjunction of conditionals. There are two natural candidates here, the first being:

The Biquation $p(B \leftrightarrow A)=p((A \rightarrow B) \wedge(B \rightarrow A))$ for any $A, B$.

But there is also the alternative:

The Other Biquation $p(B \leftrightarrow A)=p((A \rightarrow B) \wedge(\neg A \rightarrow \neg B))$ for any $A, B$.

Some hold that indicative conditionals do not contrapose, in which case The Other Biquation is not equivalent to The Biquation. So we must consider both proposals.

A word of caution: neither The Biquation nor The Other Biquation should be taken to presuppose an analysis of the biconditional as a conjunction of conditionals. These equalities merely say that the probability of a biconditional is always the same as the probability of the corresponding conjunction of conditionals. It does not follow that the biconditional is shorthand for, or is logically or analytically equivalent to, the corresponding conjunction.

Both The Biquation and The Other Biquation lead to absurdity. The conjunction of The Equation and The Biquation entails that $p(B \leftrightarrow A)=$ $p(A \wedge B)$ for any $A, B$. And the conjunction of The Equation and The Other Biquation entails that $p(B \leftrightarrow A)=0$ for any $A, B$. (See the Appendix for proofs.) As with Lewis's triviality result, the proofs here turn on being able to assign probabilities to biconditionals, so NTV about biconditionals can again be used to avoid absurdity. If $B \leftrightarrow A$ does not express a proposition, we cannot treat $p(B \leftrightarrow A)$ as the probability of a proposition to which the usual laws of probability apply. So these triviality results support NTV about biconditionals if Lewis's result supports NTV about conditionals.

The Biquation and The Other Biquation are plausible assumptions from a naive standpoint, but they hit a snag in the context of the current dialectic. We are trying to show that NTVers about $\rightarrow$ should be NTVist about $\leftrightarrow$ too. But, from the point of view of the NTVer about $\rightarrow$, assumptions like The Biquation and The Other Biquation are problematic, since they attribute probabilities to embeddings of $\rightarrow$. The crux of the NTVer's argument from Lewis-triviality is that such attributions are to be regarded with suspicion, so she will likely reject assumptions like The (Other) Biquation. This snag motivates approaching $\leftrightarrow$-triviality from a second angle.

All one really needs to run the triviality proof for $\leftrightarrow$ is an assumption about the probability of a biconditional when the probability of a corresponding conditional is extreme (either 0 or 1 ). For example, rather than assume The Biquation, we can assume merely that

$$
p(B \leftrightarrow A)= \begin{cases}0 & \text { if } p(A \rightarrow B)=0, \\ p(B \rightarrow A) & \text { if } p(A \rightarrow B)=1 .\end{cases}
$$


Similarly, rather than assume The Other Biquation, we can just assume that

$$
p(B \leftrightarrow A)= \begin{cases}0 & \text { if } p(A \rightarrow B)=0, \\ p(\neg A \rightarrow \neg B) & \text { if } p(A \rightarrow B)=1 .\end{cases}
$$

These assumptions yield the same triviality results as The Biquation and The Other Biquation respectively. (Again, see the Appendix for proofs.)

These weaker assumptions can be motivated much like The (Other) Biquation was motivated, but without appealing to embeddings that are problematic from the NTV point of view. A driving thought behind both pairs of assumptions is that one's confidence in a biconditional should depend on, or at least be linked to, one's confidence in the corresponding conditionals. For example, it would be strange to be highly confident that $B \leftrightarrow A$ when one lacks confidence in both $A \rightarrow B$ and $B \rightarrow A$ (assuming for the moment that the relevant conditionals are $B \rightarrow A$ and $A \rightarrow B$ ). But we needn't assume that this connection obeys the rules of the probability calculus for $\wedge$ in general. All we need to assume is that the extreme cases work as one would naturally expect. In the extreme case where there is absolutely no chance that $A \rightarrow B$, having any confidence at all in $B \leftrightarrow A$ seems unreasonable, so $p(B \leftrightarrow A)=0$. And in the extreme case where $A \rightarrow B$ is absolutely certain, one's only doubts about $B \leftrightarrow A$ should be one's doubts about $B \rightarrow A$, so $p(B \leftrightarrow A)=p(B \rightarrow A)$. So even if the NTVer is reluctant to assign probabilities to conjunctions of conditionals for theoretical reasons, the pre-theoretic appeal of The Biquation can still be translated into motivation for Eq. 4. And, similarly, the pre-theoretic appeal of The Other Biquation can be translated into motivation for Eq. 5.

Standoffs have also been taken to support NTV about conditionals [6]. Standoffs are cases where two subjects believe contrary conditionals, yet neither appears to believe falsely. Here is an example from Bennett [2, p. 85]. Top Gate holds back a lake from a channel that splits in two directions, east and west. There are two levers, East Lever and West Lever, controlling which way the water runs when Top Gate is open. If East Lever is down, any water coming down the channel runs east, and similarly for West Lever. If both levers are down, Top Gate cannot be opened. One observer sees that West Lever is down and believes The water will run west if Top Gate opens. Another sees that East Lever is down and thinks The water will run east if Top Gate opens. By conditional non-contradiction, they cannot both believe truly, and the symmetry of the situation forbids saying that one believes truly and the other falsely. Could they both believe falsely? Then they would have to be mistaken about some relevant fact, which it seems they are not: each draws her conclusion from true premises by good reasoning, overlooking nothing and possibly even suspecting the complete truth about the situation. Apparently what these agents believe is neither true nor false, as NTV predicts.

This argument extends to biconditionals without changing the example. Because the water cannot run either direction without Top Gate opening, each observer should believe that the water will run in her direction only if Top Gate opens. So the observer in the east is entitled to believe The water will run east 
if and only if Top Gate opens, and similarly for the observer in the west. The two observers are thus entitled to contrary biconditionals, and assigning truth values is just as fraught as before. NTV escapes the problem, however, and thus garners support.

There are many other arguments for NTV about conditionals, and we suspect that many of them can be similarly extended to biconditionals. In general, we conjecture, only minimal assumptions need to be added, and those assumptions either follow naturally from the original assumptions or else can be motivated in much the same way as the original assumptions. We will briefly outline two more examples to illustrate.

Edgington's [5, pp. 278-80] argument for NTV about conditionals extends quite naturally to biconditionals. Adapting Bennett's presentation of the argument to the case of biconditionals, we begin with the premises:

(P1) Being certain that $(A \wedge B) \vee(\neg A \wedge \neg B)$, without being certain of either $A \wedge B$ or $\neg A \wedge \neg B$, is sufficient for being certain that $B \leftrightarrow A$.

(P2) It is not necessarily irrational to disbelieve both $A$ and $B$, yet also disbelieve $B \leftrightarrow A$.

If biconditionals have truth values, (P2) tells us that it is possible for $B \leftrightarrow A$ to be false while $A$ and $B$ are both false, i.e. while $(A \wedge B) \vee(\neg A \wedge \neg B)$ is true. But then certainty in $(A \wedge B) \vee(\neg A \wedge \neg B)$ could not entitle one to certainty in $B \leftrightarrow A$, as (P1) says. The contradiction is blocked by NTV, however, since it forbids concluding from (P2) that $B \leftrightarrow A$ can be false while $A$ and $B$ are both false, since $B \leftrightarrow A$ cannot be false at all.

The following argument is an adaptation of Bennett's presentation of Bradley's [4] argument. ${ }^{6}$ Our assumptions are:

(P3) For any propositions $Q, R$, and $S$ such that neither $Q$ nor $R$ entails $S$, it can be that $p(Q)>0, p(R)>0$, and $p(S)=0$.

(P4) It is never the case that $p(A)>0$ and $p(B \leftrightarrow A)>0$ and yet $p(B)=0$.

Typically neither $A$ nor $B \leftrightarrow A$ entails $B$, so (P3) says it should be possible to assign non-zero probability to $A$ and to $B \leftrightarrow A$ but zero probability to $B$. But this is precisely what (P4) forbids. Once again, NTV can block the contradiction by saying that (P3) does not apply to $B \leftrightarrow A$, since it does not express a (truth-evaluable) proposition.

\section{Embeddability}

Oddly and unexpectedly, if and only if embeds successfully in those conditionals where iffthen does not. Consider the following parallel to Gibbard's (1):

(6) If Kripke was there if and only if Strawson was there, Anscombe was there.

\footnotetext{
${ }^{6}$ The adaptation is trivial in a way: all one must do is verify that Bradley's (P4) holds for $\leftrightarrow$ as well as for $\rightarrow$. Nevertheless, we reproduce the argument in compact form here for completeness.
} 
(6) is unwieldy. (6) is awkward. Few people would ever put what (6) expresses by uttering (6). Nonetheless, it is not difficult to interpret. ${ }^{7}$ Notice also that (6)'s cognate:

(7) If Kripke was there just in case Strawson was there, Anscombe was there. is equally interpretable.

It is no fluke that (6) is better than (1). Here are a few more pairs to illustrate:

(8) ?If Jimmy has a cat if he has a dog, then he has a parrot.

(9) If Jimmy has a cat if and only if he has a dog, then he has a parrot.

and

(10) ?If Mary has tuberculosis if the test paper shows green, they are testing for the lethal kind.

(11) If Mary has tuberculosis if and only if the test paper shows green, they are testing for the lethal kind.

In each of these pairs, the latter example is significantly more acceptable than the former. ${ }^{8}$

To further contrast (6) with (1), we can consider what inferences one would be willing to draw from each with collateral information. Consider:

A: If Kripke was there if Strawson was there, Anscombe was there.

B: Anscombe wasn't there.

It is difficult to know what to conclude from these premises, and the difficulty is exacerbated by an old problem noted by theorists of conditionals: that a negation over a conditional tends to get read as a negation of the consequent [5, p. 283]. In contrast, however, consider:

$\mathrm{A}^{\prime}$ : If Kripke was there if and only if Strawson was there, Anscombe was there.

B: Anscombe wasn't there.

It is entirely reasonable here to conclude that it's not the case that Kripke was there if and only if Strawson was.

It is tempting to dismiss (6) on the grounds that the biconditional is a term of art we learn in logic class when we learn the material biconditional. But even if if and only if is not a part of colloquial English, it is a part of academic English,

\footnotetext{
${ }^{7}$ Both authors have given this paper several times and run the sample sentences by philosophers, linguists, and laypeople; the contrast between (1) and (6) was robust in all cases.

${ }^{8}$ Most of our audience members found that neither (8) nor (10) was quite as bad as (1). But neither was deemed acceptable either, whereas (9) and (11) were. The difficulty finding examples as bad as (1) corroborates our view (see Section 4 below) that (1)'s badness is not due to semantic defectiveness, but something more idiosyncratic and superficial.
} 
and it is false that it is academic English for the material biconditional. The material biconditional:

(12) The Toronto Maple Leafs won the 1978 Stanley Cup $\equiv$ the price of milk was $\$ 99$ per liter that year.

is true because both sides are false, but the English biconditional:

(13) The Toronto Maple Leafs won the 1978 Stanley Cup if and only if the price of milk was $\$ 99$ per liter that year.

is intuitively false. Indeed, the obvious conjecture that a biconditional $B$ if and only if $A$ is intuitively true only if both $B$ if $A$ and $B$ only if $A$ are, is very reliable. So it is no surprise that $\leftrightarrow$ cannot be analyzed as $\equiv$. At least, analyzing $\leftrightarrow$ as $\equiv$ will be no more successful than analyzing $\rightarrow$ as $\supset$, and the NTVer presumably finds the latter analysis unsatisfactory.

\section{Discussion}

We have argued that biconditionals embed in conditionals where conditionals do not, yet NTVers about conditionals should also be NTVers about biconditionals. We think this is bad news for the NTV explanation of the badness of Gibbard's (1). We will briefly suggest an alternative explanation in a moment, but we want address two worries first.

The first worry is that we were too quick in concluding that NTVers about if/then should also be NTVers about if and only if. Sometimes a reason to think $P$ and $Q$ lack truth values doesn't translate into a reason to think $P \wedge Q$ lacks a truth value. For example:

(14) Holmes was tall

might lack a truth value because we can assign it true or false consistent with all that Doyle wrote. And likewise for:

(15) Holmes was not tall.

Yet:

(16) Holmes was tall and Holmes was not tall.

is surely false. So just because something is a reason for NTV about iffthen and about only if does not mean it is a reason for NTV about if and only if. ${ }^{9}$

To assuage this worry, we just have to look at the specifics of our arguments for NTV about if and only if. In the Holmes example, an argument for NTV about $P$ and $Q$ has true premises, but the analogous premise in the corresponding argument for NTV about $P \wedge Q$ is false. To ensure that our arguments do not make the same mistake, we just have to check that

\footnotetext{
${ }^{9}$ We are grateful to Alan Hájek here.
} 
the new premises are true-or, at least, that the reasons NTVers have for endorsing the premises of their arguments are equally reasons for endorsing the premises of our arguments. And this has been done. In our extension of the argument from Lewis's triviality result, for example, we argued that the additional assumptions Eqs. 4 and 5 should be amenable to the proponent of the original argument. Of course, the NTVer could allow that the premises of our arguments are true and insist that the reasoning is defeated in the case of if and only if in a way that the reasoning about if/then is not. But we do not see any reason to think this is so, and the burden is on NTVers to show that it is.

The second worry is that one might accept NTV about both conditionals and biconditionals, yet expect them to display different patterns of embedability. After all, sentences like (14) might lack truth values, but we don't expect any trouble embedding them. Merely lacking a truth value isn't enough for a prediction of infelicitous embedding. ${ }^{10}$

But NTV about sentences like (14) has a very different source: it is underdetermination of truth value, as opposed to unavailability of semantic type for assertion, that is the source of truth-valuelessness. Since the sentence is truth-apt, we should expect a different profile of embeddings comparable to whatever we give to underdetermination of truth value in general. For example, supervaluationism and some three valued logics predict that (16) will express a falsehood, as will

(17) John believes that Holmes is tall.

Nothing comparable happens with conditionals and biconditionals, where the source of truth-valuelessness is the hypothesis that they don't express propositions at all.

We conclude that the badness of (1) is not to be explained by appeal to NTV. We will close by mentioning a possible alternative.

There are cases of perfectly meaningful sentences that are nevertheless hard to interpret, most famously "garden path" sentences like:

(18) The horse raced past the barn fell. ${ }^{11}$

Sentence (18) sounds awfully bad, until understood as:

(19) The horse that was raced past the barn fell.

A different sort of example is Miller and Chomsky's [11]:

(20) The rat the cat the dog chased ate died.

\footnotetext{
${ }^{10}$ Here again we are grateful to Alan Hájek.

${ }^{11}$ The example is from [3].
} 
Sentence (20) is generally regarded as syntactically and semantically wellformed, yet intuitively unacceptable due to limitations of the human parser $[8] .{ }^{12}$

Perhaps there is something similar at work in cases like (1). This suggestion gains support from the fact that it is somewhat difficult to understand similar sentences, ${ }^{13}$ such as:

(21) ?Because Mary came because Sheila came, Steve came.

Compare (21) with:

(22) Because Mary came because and only because Sheila came, Steve came.

Sentence (22) is not easily understood but, despite its clumsy structuring, it is understandable. These observations suggest that the badness of (1) is better explained by parsing difficulties than by semantic defectiveness. ${ }^{14}$

One might object that parsing difficulties could not explain the difference between Gibbard's (1) and our (6), since (1) is syntactically simpler. But syntactic simplicity does not closely track ease of parsing. (18) is not easily parsed, yet:

(23) The horse slaughtered behind the barn fell.

is syntactically comparable and is easily parsed. The reason is clear: slaughtered doesn't plausibly take an agentive argument in this context since it would be transitive and there is no patient to play the role. The human parser is guided and misguided by semantic as well syntactic complexity.

A better question, we think, is why (1) would trip up a parser while (2) fails to cause similar problems. We aren't sure. It's tempting to think that repetition of the same verb in the same structure does the trick, but this depends on just how uninterpretable (21) is. Our best guess is that left-dislocated clauses that begin with connectives like if/then or because cause scope trouble when one tries to reconstruct them. ${ }^{15}$

Acknowledgements We are grateful to John Divers, Branden Fitelson, Michael Glanzberg, Alan Hájek, John Hawthorne, David Horacek, Carrie Jenkins, Phil Kremer, Jennifer Nagel, Daniel Nolan, Charlie Snow, Robert Stalnaker, Robbie Williams, audiences at the University of Nottingham, the University of Leeds, and the 2008 Formal Epistemology Festival, and several anonymous referees for helpful discussion and feedback.

\footnotetext{
${ }^{12}$ We are grateful to Jennifer Nagel here.

${ }^{13}$ We are grateful to John Hawthorne here.

${ }^{14}$ We leave open whether cases like (1) are the same in nature as (18) or (20), or whether they present the parser with a different sort of challenge. (See [12, p. 207] for one who suggests that (1) is problematic for the same reason as (20).) We also leave open whether the difficulty parsing (1) is ultimately syntactic or semantic in nature.

${ }^{15} \mathrm{Or}$, in the case where they are stacked, the parser is confused by following a connective, looking for a proposition with a connective that later has to be reconstructed at LF.
} 
Open Access This article is distributed under the terms of the Creative Commons Attribution Noncommercial License which permits any noncommercial use, distribution, and reproduction in any medium, provided the original author(s) and source are credited.

\section{Appendix: Triviality Results for Biconditionals}

The Biquation entails Eq. 4 and The Other Biquation entails Eq. 5, so it will suffice to show that the weaker assumptions Eqs. 4 and 5 each lead to a triviality result.

Claim 1 Given The Equation and Eq. 4, $p(B \leftrightarrow A)=p(A \wedge B)$ for any $A, B$.

Proof Assume The Equation and Eq. 4, and let $p_{\phi}(\cdot)$ be the probability function obtained from $p$ by conditionalizing on $\phi$.

Lewis showed that, given The Equation, the probability of a conditional is always the probability of its consequent, from which it follows immediately that $p_{B}(A \rightarrow B)=1$. By Eq. 4 then,

$$
p_{B}(B \leftrightarrow A)=p_{B}(B \rightarrow A) .
$$

And by The Equation,

$$
\begin{aligned}
p_{B}(B \rightarrow A) & =p_{B}(A \mid B) \\
& =p(A \mid B) .
\end{aligned}
$$

So

$$
p_{B}(B \leftrightarrow A)=p(A \mid B) .
$$

From Lewis's result we also have $p_{\neg B}(A \rightarrow B)=0$, so Eq. 4 gives us

$$
p_{\neg B}(B \leftrightarrow A)=0 .
$$

Applying the law of total probability then, we have

$$
\begin{aligned}
p(B \leftrightarrow A) & =p(B \leftrightarrow A \mid B) p(B)+p(B \leftrightarrow A \mid \neg B) p(\neg B) \\
& =p_{B}(B \leftrightarrow A) p(B)+p_{\neg B}(B \leftrightarrow A) p(\neg B) \\
& =p(A \mid B) p(B) \\
& =p(A \wedge B) .
\end{aligned}
$$

Claim 2 Given The Equation and Eq. 5, $p(B \leftrightarrow A)=0$ for any $A, B$.

Proof The proof is similar to the proof of Claim 1, except that now both $p_{B}(B \leftrightarrow A)$ and $p_{\neg B}(B \leftrightarrow A)$ are 0 . The reason for $p_{\neg B}(B \leftrightarrow A)=0$ is the same as before: Lewis and The Equation tell us that $p_{\neg B}(A \rightarrow B)=0$, so Eq. 5 tells us 
that $p_{\neg B}(B \leftrightarrow A)=0$. As for $p_{B}(B \leftrightarrow A)$, The Equation gives us $p_{B}(A \rightarrow B)=1$, so Eq. 5 yields

$$
p_{B}(B \leftrightarrow A)=p_{B}(\neg A \rightarrow \neg B) .
$$

And since The Equation tells us $p_{B}(\neg A \rightarrow \neg B)=0$, we have

$$
p_{B}(B \leftrightarrow A)=0 .
$$

Thus, when we apply the law of total probability to $p(B \leftrightarrow A)$, both summands are 0 :

$$
\begin{aligned}
p(B \leftrightarrow A) & =p(B \leftrightarrow A \mid B) p(B)+p(B \leftrightarrow A \mid \neg B) p(\neg B) \\
& =p_{B}(B \leftrightarrow A) p(B)+p_{\neg B}(B \leftrightarrow A) p(\neg B) \\
& =0 .
\end{aligned}
$$

\section{References}

1. Arló-Costa, H. (1999). Belief revision conditionals: Basic iterated systems. Annals of Pure and Applied Logic, 96(1), 3-28.

2. Bennett, J. (2003). A Philosophical Guide to Conditionals. Oxford University Press.

3. Bever, T. (1970). The cognitive basis for linguistic structures. In Hayes, J. R. (Ed.), Cognition and the Development of Language, (pp. 279-362). New York: Wiley.

4. Bradley, R. (2000). A preservation condition for conditionals. Analysis, 60(3), 219-22.

5. Edgington, D. (1995). On conditionals. Mind, 104(414), 235-329.

6. Gibbard, A. (1981). Two recent theories of conditionals. In Harper, W. L., Stalnaker, R., Pearce, G. (Eds.), Ifs, (pp. 211-247). Reidel.

7. Hansson, S. O. (1992). A dyadic representation of belief. In Gärdenfors, P. (Ed.), Belief Revision, (pp. 89-121). Cambridge University Press.

8. Hudson, R. (1996). The difficulty of (so-called) self-embedded structures. UCL Working Papers in Linguistics, 8(1), 283-314.

9. Levi, I. (1996). For the Sake of the Argument: Ramsey Test Conditionals, Inductive Inference, and Nonmonotonic Reasoning. Cambridge University Press.

10. Lewis, D. (1976). Probabilities of conditionals and conditional probabilities. The Philosophical

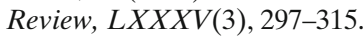

11. Miller, G., \& Chomsky, N. (1963). Finitary models of language users. In Handbook of Mathematical Psychology, (Vol. 2, pp. 419-493). New York: Wiley.

12. Pinker, S. (1994). The Language Instinct. Harmondsworth:Penguin. 